\title{
Polarization effects in non-relativistic ep scattering
}

\author{
A.I. Milstein, S.G. Salnikov, and V.M.Strakhovenko \\ Budker Institute of Nuclear Physics, 630090 Novosibirsk, Russia
}

(Dated: August 17, 2021)

\begin{abstract}
The cross section which addresses the spin-flip transitions of a proton (antiproton) interacting with a polarized non-relativistic electron or positron is calculated analytically. In the case of attraction, this cross section is greatly enhanced for sufficiently small relative velocities as compared to the result obtained in the Born approximation. However, it is still very small, so that the beam polarization time turns out to be enormously large for the parameters of $e^{ \pm}$beams available now. This practically rules out a use of such beams to polarize stored antiprotons or protons.
\end{abstract}

PACS numbers: 13.88.+e, 29.20.Dh, 29.27.Hj 


\section{INTRODUCTION}

An extensive physical program with polarized antiprotons has been proposed recently by the PAX Collaboration [1]. This program has initiated a discussion of various methods to polarize stored antiprotons (see [1, 2] and literature therein). One option, which was considered, is to use for this purpose the interaction with a polarized electron (positron) beam. When the relative velocity $v$ of two beams is sufficiently small, the scattered antiprotons remain in the beam. In this case, the polarization buildup is completely due to the spin-flip transitions, as it was explained in Refs. [3, 4]. The corresponding cross section was estimated in Ref. [3] in the Born approximation and turned out to be too small (about $10^{-3} \mathrm{mb}$ ) to provide a noticeable polarization in a reasonable time.

At very small velocities, when $\alpha / v \gtrsim 1$ ( $\alpha$ is the fine-structure constant and $\hbar=c=1$ ), the Born approximation becomes invalid. It is well known that for such velocities the cross section is modified due to a corresponding change of the $e^{+}\left(e^{-}\right)$flux near the origin. A magnitude of the effect can be characterized (see, e.g. [5]) by the parameter $C(\xi)$ being the squared ratio of the Coulomb and free wave function at the origin:

$$
C(\xi)=\frac{2 \pi \xi}{\exp (2 \pi \xi)-1}
$$

here $\xi=e_{1} e_{2} / v$, so that $\xi=\alpha / v$ for repulsion $\left(e^{+} p\right.$ or $e^{-} \bar{p}$ interaction $)$ and $\xi=-\alpha / v$ for attraction $\left(e^{-} p\right.$ or $e^{+} \bar{p}$ interaction). At $|\xi| \gg 1$, the quantity $C(\xi)$ is large only in the case of attraction where $C(\xi)=2 \pi|\xi|$. Since the cross section contains terms $\propto C^{2}(\xi)$, we can figure on the enhancement in $e^{-} p$ or $e^{+} \bar{p}$ interaction of the order $(2 \pi \xi)^{2}$ as compared with the Born approximation.

Recently, the enhancement that exceeds $C^{2}(\xi)$ by many orders of magnitude was claimed in Refs. [6, 7]. Correspondingly, the conclusion was drawn in [6] that antiprotons can be easily polarized by interaction with a polarized positron beam. The crucial point of the calculation, estimation of the radial integrals, was performed in [6, 7] numerically.

To shed light on the problem, we perform in the present paper an analytical calculation of the cross section, which addresses the spin-flip transitions of an antiproton (proton) interacting with a polarized non-relativistic positron or electron. Just $C^{2}(\xi)$ is obtained as the enhancement factor. It is big enough, but the resulting cross section is still very small (less than $1 \mathrm{mb}$ at $v=0.0019$ used for estimations in [6]). Using this cross section, we analyze 
the kinetics of the polarization buildup. The polarization time turns out to be enormously large for the parameters of $e^{+}$or $e^{-}$beams available now. To give a feeling of how far we are from the practical use of the effect, we note that the scale of days for the polarization time at $v=0.0019$ can be achieved when the positron beam density becomes higher than $5 \cdot 10^{16} \mathrm{~cm}^{-3}$.

\section{CROSS SECTION}

We assume that $v \ll 1$, where $v$ is the relative velocity of an electron and a proton. Then one can neglect recoil effects for the proton and consider it as a source of the Coulomb field. Thus, we calculate all spin effects with the help of perturbation theory using the exact Coulomb non-relativistic wave functions. A part of the Hamiltonian of the $e p$ interaction dependent on the proton spin reads [8]

$$
H=\frac{\alpha \mu_{0}}{m_{e} m_{p}}\left\{\frac{3\left(\boldsymbol{n} \cdot \boldsymbol{s}_{1}\right)\left(\boldsymbol{n} \cdot \boldsymbol{s}_{2}\right)-\left(\boldsymbol{s}_{1} \cdot \boldsymbol{s}_{2}\right)}{r^{3}}+\frac{8 \pi}{3} \delta(\boldsymbol{r})\left(\boldsymbol{s}_{1} \cdot \boldsymbol{s}_{2}\right)+\frac{\boldsymbol{L} \cdot \boldsymbol{s}_{1}}{r^{3}}\right\},
$$

where $\boldsymbol{s}_{1}$ and $\boldsymbol{s}_{2}$ are the spin operators of a proton and an electron, respectively, $\boldsymbol{L}$ is the angular momentum operator of an electron, and $\mu_{0}=2.79$ is the dimensionless proton magnetic moment in units of the nuclear magneton. We introduce the spinors $\phi_{\sigma}$ and $\chi_{\lambda}$ describing the proton and electron spin states, respectively. Let the initial electron be polarized along the unit vector $\boldsymbol{\zeta}_{e}$ and the initial proton along $\boldsymbol{\zeta}_{p}$, so that $2 \phi_{\sigma}^{+} \boldsymbol{s}_{1} \phi_{\sigma}=\boldsymbol{\zeta}_{p}$ and $2 \chi_{\lambda_{i}}^{+} s_{2} \chi_{\lambda_{i}}=\boldsymbol{\zeta}_{e}$. Then the cross section of scattering with the spin-flip transition of the proton has the form

$$
d \sigma=\frac{m_{e}^{2} d \Omega_{2}}{(2 \pi)^{2}} \sum_{\lambda_{f}}|M|^{2}, \quad M=\int d \boldsymbol{r} \phi_{-\sigma}^{+} \chi_{\lambda_{f}}^{+} \psi_{\boldsymbol{p}_{2}}^{*(-)}(\boldsymbol{r}) H \psi_{\boldsymbol{p}_{1}}^{(+)}(\boldsymbol{r}) \phi_{\sigma} \chi_{\lambda_{i}}
$$

where $\boldsymbol{p}_{1}$ and $\boldsymbol{p}_{2}$ are the momenta of the initial and final electron, $\Omega_{2}$ is the solid angle of the

vector $\boldsymbol{p}_{2}, \psi_{\boldsymbol{p}_{1}}^{(+)}(\boldsymbol{r})$ is the wave function of the initial electron containing at large distances a plain wave and the divergent spherical wave, $\psi_{\boldsymbol{p}_{2}}^{(-)}(\boldsymbol{r})$ is the wave function of the final electron containing at large distances a plain wave and the convergent spherical wave. These wave functions read

$$
\begin{aligned}
& \psi_{\boldsymbol{p}_{2}}^{(-)}(\boldsymbol{r})=\frac{1}{2 p} \sum_{l=0}^{\infty}(2 l+1) i^{l} \mathrm{e}^{-i \delta_{l}} P_{l}\left(\boldsymbol{k}_{2} \cdot \boldsymbol{n}\right) R_{p, l}(r), \\
& \psi_{\boldsymbol{p}_{1}}^{(+)}(\boldsymbol{r})=\frac{1}{2 p} \sum_{l=0}^{\infty}(2 l+1) i^{l} \mathrm{e}^{i \delta_{l}} P_{l}\left(\boldsymbol{k}_{1} \cdot \boldsymbol{n}\right) R_{p, l}(r) .
\end{aligned}
$$


Here $\boldsymbol{k}_{1,2}=\boldsymbol{p}_{1,2} / p, \boldsymbol{n}=\boldsymbol{r} / r, \delta_{l}$ and $R_{p, l}(r)$ are, respectively, the phase shifts and radial wave functions of an electron in a Coulomb field, $P_{l}(x)$ are the Legendre polynomials. Substituting these wave functions into Eq. (3) we obtain the matrix element in the form

$$
\begin{aligned}
& M=\frac{\alpha \mu_{0}}{4 p^{2} m_{e} m_{p}}\left\{\frac{8 \pi}{3}\left(\boldsymbol{S}_{1} \cdot \boldsymbol{S}_{2}\right) \mathrm{e}^{2 i \delta_{0}} R_{p, 0}^{2}(0)+\sum_{l=1}^{\infty}(2 l+1)^{2} \mathrm{e}^{2 i \delta_{l}} \int_{0}^{\infty} \frac{d r}{r} R_{p, l}^{2}(r)\right. \\
& \times \int d \boldsymbol{n} P_{l}\left(\boldsymbol{k}_{2} \cdot \boldsymbol{n}\right)\left[3\left(\boldsymbol{n} \cdot \boldsymbol{S}_{1}\right)\left(\boldsymbol{n} \cdot \boldsymbol{S}_{2}\right)-\left(\boldsymbol{S}_{1} \cdot \boldsymbol{S}_{2}\right)+\delta_{\lambda_{f}, \lambda_{i}} \boldsymbol{L} \cdot \boldsymbol{S}_{1}\right] P_{l}\left(\boldsymbol{k}_{1} \cdot \boldsymbol{n}\right) \\
& -\sum_{l=0}^{\infty}(2 l+1)(2 l+5) \mathrm{e}^{i\left(\delta_{l}+\delta_{l+2}\right)} \int_{0}^{\infty} \frac{d r}{r} R_{p, l}(r) R_{p, l+2}(r) \\
& \times \int d \boldsymbol{n}\left[P_{l}\left(\boldsymbol{k}_{2} \cdot \boldsymbol{n}\right) P_{l+2}\left(\boldsymbol{k}_{1} \cdot \boldsymbol{n}\right)+P_{l}\left(\boldsymbol{k}_{1} \cdot \boldsymbol{n}\right) P_{l+2}\left(\boldsymbol{k}_{2} \cdot \boldsymbol{n}\right)\right] \\
& \left.\times\left[3\left(\boldsymbol{n} \cdot \boldsymbol{S}_{1}\right)\left(\boldsymbol{n} \cdot \boldsymbol{S}_{2}\right)-\left(\boldsymbol{S}_{1} \cdot \boldsymbol{S}_{2}\right)\right]\right\},
\end{aligned}
$$

where $\boldsymbol{S}_{1}=\phi_{-\sigma}^{+} \boldsymbol{s}_{1} \phi_{\sigma}$ and $\boldsymbol{S}_{2}=\chi_{\lambda_{f}}^{+} \boldsymbol{s}_{2} \chi_{\lambda_{i}}$.

In what follows we assume that in the rest frame of a proton beam the velocities $\boldsymbol{v}$ of electrons are randomly distributed over their directions. Therefore we average the cross section over the direction of the vector $\boldsymbol{k}_{1}$. It is convenient to perform integrations in the following order. We integrate $|M|^{2}$ first over $\boldsymbol{k}_{1}$ and $\boldsymbol{k}_{2}$ and then over the angles of $\boldsymbol{n}$ using the relations

$$
\begin{aligned}
& \int d \boldsymbol{k} P_{l}(\boldsymbol{k} \cdot \boldsymbol{n}) P_{l}\left(\boldsymbol{k} \cdot \boldsymbol{n}^{\prime}\right)=\frac{4 \pi}{2 l+1} P_{l}\left(\boldsymbol{n} \cdot \boldsymbol{n}^{\prime}\right), \\
& \iint d \boldsymbol{n}_{1} d \boldsymbol{n}_{2} P_{l}\left(\boldsymbol{n}_{1} \cdot \boldsymbol{n}_{2}\right) P_{l^{\prime}}\left(\boldsymbol{n}_{1} \cdot \boldsymbol{n}_{2}\right)\left(3 n_{1}^{i} n_{1}^{j}-\delta^{i j}\right)\left(3 n_{2}^{a} n_{2}^{b}-\delta^{a b}\right) \\
& =\frac{8 \pi^{2}}{5}\left[3\left(\delta^{i a} \delta^{j b}+\delta^{i b} \delta^{j a}\right)-2 \delta^{i j} \delta^{a b}\right] \int_{-1}^{1} d x P_{2}(x) P_{l}(x) P_{l^{\prime}}(x), \\
& \iint d \boldsymbol{n}_{1} d \boldsymbol{n}_{2}\left[\left(\boldsymbol{S}_{1} \cdot \boldsymbol{L}_{1}\right) P_{l}\left(\boldsymbol{n}_{1} \cdot \boldsymbol{n}_{2}\right)\right]\left[\left(\boldsymbol{S}_{1}^{*} \cdot \boldsymbol{L}_{2}\right) P_{l}\left(\boldsymbol{n}_{1} \cdot \boldsymbol{n}_{2}\right)\right]=\frac{(4 \pi)^{2}}{3}\left(\boldsymbol{S}_{1} \cdot \boldsymbol{S}_{1}^{*}\right) \frac{l(l+1)}{2 l+1} .
\end{aligned}
$$

As a result we have

$$
\begin{aligned}
& \sigma=\pi\left(\frac{\alpha \mu_{0}}{p^{2} m_{p}}\right)^{2} \sum_{\lambda_{f}}\left\{\frac{1}{5}\left[3\left(\left(\boldsymbol{S}_{1} \cdot \boldsymbol{S}_{1}^{*}\right)\left(\boldsymbol{S}_{2} \cdot \boldsymbol{S}_{2}^{*}\right)+\left|\left(\boldsymbol{S}_{1} \cdot \boldsymbol{S}_{2}^{*}\right)\right|^{2}\right)-2\left|\left(\boldsymbol{S}_{1} \cdot \boldsymbol{S}_{2}\right)\right|^{2}\right]\right. \\
& \times\left[\sum_{l=1}^{\infty} \frac{l(l+1)(2 l+1)}{(2 l+3)(2 l-1)} \mathcal{F}_{l}^{2}+\sum_{l=0}^{\infty} \frac{3(l+1)(l+2)}{(2 l+3)} \mathcal{G}_{l}^{2}\right]+\left|\frac{2}{3}\left(\boldsymbol{S}_{1} \cdot \boldsymbol{S}_{2}\right) R_{p, 0}^{2}(0)\right|^{2} \\
& \left.+\frac{1}{3} \delta_{\lambda_{f}, \lambda_{i}}\left(\boldsymbol{S}_{1} \cdot \boldsymbol{S}_{1}^{*}\right) \sum_{l=1}^{\infty} l(l+1)(2 l+1) \mathcal{F}_{l}^{2}\right\}
\end{aligned}
$$


with

$$
\mathcal{F}_{l}=\int_{0}^{\infty} \frac{d r}{r} R_{p, l}^{2}(r), \quad \mathcal{G}_{l}=\int_{0}^{\infty} \frac{d r}{r} R_{p, l}(r) R_{p, l+2}(r)
$$

Calculating the vectors $\boldsymbol{S}_{1}$ and $\boldsymbol{S}_{2}$ and taking the sum over $\lambda_{f}$, we finally arrive at

$$
\begin{aligned}
& \sigma=\pi\left(\frac{\alpha \mu_{0}}{p^{2} m_{p}}\right)^{2}\left\{\frac{1}{8}\left(2+\boldsymbol{\zeta}_{e} \cdot \boldsymbol{\zeta}_{p}\right)\left[\sum_{l=1}^{\infty} \frac{l(l+1)(2 l+1)}{(2 l+3)(2 l-1)} \mathcal{F}_{l}^{2}+\sum_{l=0}^{\infty} \frac{3(l+1)(l+2)}{(2 l+3)} \mathcal{G}_{l}^{2}\right]\right. \\
& \left.+\frac{1}{18}\left(1-\boldsymbol{\zeta}_{e} \cdot \boldsymbol{\zeta}_{p}\right) R_{p, 0}^{4}(0)+\frac{1}{6} \sum_{l=1}^{\infty} l(l+1)(2 l+1) \mathcal{F}_{l}^{2}\right\} .
\end{aligned}
$$

A contribution of the $L S$-interaction to $\sigma$ is given by the last term in Eq. (9) which is independent of the electron spin. Therefore, it does not lead to the appearance of a hadron beam polarization but contributes to its depolarization.

\section{RADIAL INTEGRALS}

Let us consider the functions $\mathcal{F}_{l}$ and $\mathcal{G}_{l}$. We calculate them using the convenient integral representation of the electron Green function in a Coulomb field found in Ref. [9] and the relation for the wave functions of the continuous spectrum

$$
\int \frac{d \boldsymbol{k}}{4 \pi} \psi_{\boldsymbol{p}}\left(\boldsymbol{r}_{1}\right) \psi_{\boldsymbol{p}}^{*}\left(\boldsymbol{r}_{2}\right)=\frac{i \pi}{p m_{e}} \delta G\left(\boldsymbol{r}_{1}, \boldsymbol{r}_{2} \mid E\right),
$$

where $\boldsymbol{k}=\boldsymbol{p} / p, E=p^{2} /\left(2 m_{e}\right)$, and

$$
\delta G\left(\boldsymbol{r}_{1}, \boldsymbol{r}_{2} \mid E\right)=G\left(\boldsymbol{r}_{1}, \boldsymbol{r}_{2} \mid E+i 0\right)-G\left(\boldsymbol{r}_{1}, \boldsymbol{r}_{2} \mid E-i 0\right)
$$

is the discontinuity of the Green function on the cut. Then we obtain

$$
R_{p, l}\left(r_{1}\right) R_{p, l}\left(r_{2}\right)=\frac{2 p(-1)^{l+1}}{\sqrt{r_{1} r_{2}}} \int_{-\infty}^{+\infty} \frac{d s}{\sinh s} \exp \left[i p\left(r_{1}+r_{2}\right) \operatorname{coth} s-2 i \xi s\right] J_{2 l+1}\left(\frac{2 p \sqrt{r_{1} r_{2}}}{\sinh s}\right)
$$

where $J_{\nu}(x)$ is the Bessel function. Remember that, according to our definition Eq. (1), $\xi=-\alpha / v$ for the attractive potential and $\xi=\alpha / v$ for the repulsive one. For the square of the radial wave function we obtain from Eq. (11)

$$
R_{p, l}^{2}(r)=\frac{2 p(-1)^{l+1}}{r} \int_{-\infty}^{+\infty} \frac{d s}{\sinh s} \exp [2 i p r \operatorname{coth} s-2 i \xi s] J_{2 l+1}\left(\frac{2 p r}{\sinh s}\right) .
$$


Substituting the asymptotic form of $R_{p, l}\left(r_{2}\right)$ at small distances [5] into Eq. (11), we arrive at the following integral representation for $R_{p, l}(r)$

$$
R_{p, l}(r)=\frac{p 2^{-l}(p r)^{l}(-1)^{l+1}}{\exp (-\pi \xi / 2)|\Gamma(l+1+i \xi)|} \int_{-\infty}^{+\infty} \frac{d s}{(\sinh s)^{2 l+2}} \exp [i p r \operatorname{coth} s-2 i \xi s]
$$

where $\Gamma(x)$ is the Euler gamma function. Then we substitute Eq. (12) into the definition of the function $\mathcal{F}_{l}$ in Eq. (8) and take the integral first over $r$ and then over the parameter $s$. In order to change the order of integration we deform the contour of integration over $s$ so that it passes in the positive direction around the point $s=0$. After that all integrals can be easily taken and we obtain

$$
\mathcal{F}_{l}=\frac{(2 p)^{2}}{2 l(l+1)} f_{l}(\xi), \quad f_{l}(\xi)=\left\{1+\frac{\xi}{2 l+1}[2 \operatorname{Im} \psi(l+1+i \xi)-\pi]\right\},
$$

where $\psi(z)=d \ln \Gamma(z) / d z$. In the limit $\xi \rightarrow 0$, i.e. in the Born approximation, $f_{l}(\xi) \rightarrow 1$. The term in $f_{l}(\xi)$ linear with respect to $\xi$ is the only odd term. It comes from the contribution of the small half-circle around the point $s=0$. All the higher order terms in $\xi$ are the even functions of $\xi$. Using the relation

$$
\operatorname{Im} \psi(l+1+i \xi)=\xi \sum_{k=0}^{\infty} \frac{1}{(k+l+1)^{2}+\xi^{2}},
$$

we can present the function $f_{l}(\xi)$ as

$$
f_{l}(\xi)=\frac{1}{2 l+1}\left[C(\xi)+2 \sum_{k=1}^{l} \frac{k^{2}}{k^{2}+\xi^{2}}\right]
$$

where $C(\xi)$ is just the factor introduced in Eq. (11). So, at $|\xi| \gg 1$ the radial integral $\mathcal{F}_{l}$ is enhanced by the factor $2 \pi|\xi|$ for the attractive potential $(\xi<0)$. For the repulsive potential $(\xi>0)$, it is suppressed as $1 / \xi^{2}$, not exponentially as one can naïvely expect. The straightforward calculation with the use of Eq. (13) leads to the following expression for the function $\mathcal{G}_{l}$ Eq. (8)

$$
\mathcal{G}_{l}=\frac{(2 p)^{2}}{6|l+1+i \xi||l+2+i \xi|} .
$$

Note that $\mathcal{G}_{l}$ is an even function of $\xi$, being suppressed at $|\xi| \gg l$ as $1 / \xi^{2}$.

Substituting the calculated radial integrals Eqs. (14) and (16) into Eq. (9) and recollecting that $R_{p, 0}^{4}(0)=(2 p)^{4} C^{2}(\xi)$, we obtain the final form of the cross section

$$
\sigma=\frac{1}{2} \sigma_{0}\left\{C^{2}(\xi)\left[(3-2 \ln 2)+(\ln 2-2)\left(\boldsymbol{\zeta}_{e} \cdot \boldsymbol{\zeta}_{p}\right)\right]+\sum_{l=1}^{\infty} \frac{1}{l(l+1)(2 l+1)}\left[4 C(\xi) g_{l}\right.\right.
$$




$$
\begin{aligned}
& \left.+\left(2+\boldsymbol{\zeta}_{e} \cdot \boldsymbol{\zeta}_{p}\right)\left(\frac{3\left(C(\xi)+g_{l}\right) g_{l}}{(2 l-1)(2 l+3)}+\frac{l^{2}(l+1)^{2}}{4\left(l^{2}+\xi^{2}\right)\left((l+1)^{2}+\xi^{2}\right)}\right)\right] \\
& \left.+\sum_{l=1}^{l_{\max }} \frac{\left(2 g_{l}\right)^{2}}{l(l+1)(2 l+1)}\right\} .
\end{aligned}
$$

Here

$$
\sigma_{0}=\frac{\pi}{3}\left(\frac{2 \alpha \mu_{0}}{m_{p}}\right)^{2} \approx 0.77 \cdot 10^{-3} \mathrm{mb}, \quad g_{l}=\sum_{k=1}^{l} \frac{k^{2}}{k^{2}+\xi^{2}} .
$$

The last sum in Eq. (17) has been regularized by introducing $l_{\max } \gg 1$, as it is logarithmically divergent at large $l$. This divergence is well known (see, e.g. [8]) and addresses a singular behavior of the Born amplitude at small scattering angle. So that we can set $l_{\max }=p \rho$, where $\rho$ has a meaning of a maximal impact parameter being of the order of the transverse beam size. At $v \ll 1$, the expression Eq. (17) is valid for any values of $\xi$. At $\xi \rightarrow 0$ it goes over into

$$
\sigma_{\text {Born }}=\sigma_{0}\left[1-\frac{1}{2}\left(\boldsymbol{\zeta}_{e} \cdot \boldsymbol{\zeta}_{p}\right)+\ln \left(l_{\max }\right)\right]
$$

where the spin independent term dominates. It is evident from Eq. (17) that we can figure on some polarization effects only for the attractive potential $(\xi<0)$ at $|\xi| \gg 1$. For this case, we obtain from Eq. (17)

$$
\sigma=\sigma_{0}\left\{\frac{1}{2}(2 \pi \xi)^{2}\left[(3-2 \ln 2)+(\ln 2-2)\left(\boldsymbol{\zeta}_{e} \cdot \boldsymbol{\zeta}_{p}\right)\right]+\ln \frac{l_{\max }}{|\xi|}\right\} .
$$

Note that typically $l_{\max } \gg|\xi|$ which has been used in the derivation of Eq. (18).

\section{KINETICS OF POLARIZATION}

The kinetics of the polarization buildup due to interaction with various polarized targets was considered in detail in Ref. [3]. In the case of initially unpolarized proton beam interacting with the polarized electron beam, when the direction of the relative velocity $\boldsymbol{v}$ of electron and proton is random, the arising polarization of the proton beam is collinear with the direction of the electron beam polarization. The polarization degree of the proton beam , $P(t)$, at time $t$ reads

$$
\begin{aligned}
& P(t)=P_{e} P_{0}\left(1-\mathrm{e}^{-\Omega t}\right), \quad P_{0}=\frac{\sigma_{+-}-\sigma_{-+}}{\sigma_{+-}+\sigma_{-+}}, \\
& \Omega=f n l \sigma_{t o t} \frac{v}{V_{b}}, \quad \sigma_{t o t}=\sigma_{+-}+\sigma_{-+} .
\end{aligned}
$$


Here $f$ is a revolution frequency, $n$ is a density of the electron beam, $l$ is the length of the interaction region, $V_{b}$ is the proton beam velocity. All these quantities are defined in the lab frame. In Eq. (19), $P_{e}$ is the polarization degree of the electron beam and the cross sections $\sigma_{-+}$and $\sigma_{+-}$are obtained from Eq. (18) with $\boldsymbol{\zeta}_{p}=\boldsymbol{\zeta}_{e}$ and $\boldsymbol{\zeta}_{p}=-\boldsymbol{\zeta}_{e}$, respectively. If $P_{e}=0$ but the proton beam has some initial polarization $P(0)$, then $P(t)=P(0) \exp (-\Omega t)$, i.e. the rates of depolarization and polarization are the same.

From Eq. (18) we obtain

$$
\begin{aligned}
P_{0} & =\frac{(2-\ln 2)}{3-2 \ln 2+\ln \left(l_{\max }^{2} / \xi^{2}\right) /(2 \pi \xi)^{2}}, \\
\sigma_{\text {tot }} & =\sigma_{0}\left\{(2 \pi \xi)^{2}(3-2 \ln 2)+\ln \frac{l_{\max }^{2}}{\xi^{2}}\right\} .
\end{aligned}
$$

At $v=0.0019$ used for estimations in [6] and $\rho \approx 1 \mathrm{~cm}$, we have $P_{0} \approx 0.78$ which is big enough. However, for the same parameters, we have $\sigma_{t o t} \approx 0.75 \mathrm{mb}$ that is drastically different from the result $\sigma_{\text {tot }} \approx 4 \cdot 10^{+13}$ barn obtained in [6]. When the correct value of $\sigma_{\text {tot }}$ is used, the beam polarization time, $\tau=\Omega^{-1}$, becomes enormously large for the parameters of $e^{ \pm}$beams available now. For example, to reach the scale of days for $\tau$ at values of $v, f$, and $l$ used in [6], the positron beam density should be higher than $n \approx 5 \cdot 10^{16} \mathrm{~cm}^{-3}$. Even in the case of the permanent interaction of two beams, when $\tau=1 /\left(n v \sigma_{t o t}\right)$ is minimal, the polarization time about one day is obtained at $v=0.0019$ for the density $n \approx 3 \cdot 10^{14} \mathrm{~cm}^{-3}$.

In the present paper, the cross section which is averaged over directions of relative velocities (over $\boldsymbol{k}_{1}=\boldsymbol{p}_{1} / p$ ), is calculated. We have also obtained the cross section $\tilde{\sigma}$ without such averaging. At $\xi<0$ and $|\xi| \gg 1$, we have for $\tilde{\sigma}$

$$
\begin{aligned}
\tilde{\sigma}=\sigma & +\frac{\sigma_{0}}{8}\left\{\left[3\left(\boldsymbol{\zeta}_{p} \cdot \boldsymbol{k}_{1}\right)^{2}-1\right]\left[(2 \pi \xi)^{2}\left(\ln 2-\frac{1}{2}\right)+\ln \frac{l_{\text {max }}^{2}}{\xi^{2}}\right]\right. \\
& \left.+(2 \pi \xi)^{2}(2 \ln 2-1)\left[\left(\boldsymbol{\zeta}_{e} \cdot \boldsymbol{\zeta}_{p}\right)-3\left(\boldsymbol{\zeta}_{e} \cdot \boldsymbol{k}_{1}\right)\left(\boldsymbol{\zeta}_{p} \cdot \boldsymbol{k}_{1}\right)\right]\right\},
\end{aligned}
$$

where $\sigma$ is the cross section defined by Eq. (18). From Eq. (21), we obtain for the cross section $\tilde{\sigma}_{t o t}=\tilde{\sigma}_{+-}+\tilde{\sigma}_{-+}$in the case of the transverse $\left(\boldsymbol{\zeta}_{e} \perp \boldsymbol{k}_{1}\right)$ and longitudinal $\left(\boldsymbol{\zeta}_{e} \| \boldsymbol{k}_{1}\right)$ polarization

$$
\tilde{\sigma}_{\text {tot }}^{\perp}=\frac{3}{4} \sigma_{0}\left\{(2 \pi \xi)^{2}\left(\frac{25}{6}-3 \ln 2\right)+\ln \frac{l_{\max }^{2}}{\xi^{2}}\right\},
$$




$$
\tilde{\sigma}_{t o t}^{\|}=\frac{3}{2} \sigma_{0}\left\{(2 \pi \xi)^{2}\left(\frac{11}{6}-\ln 2\right)+\ln \frac{l_{\max }^{2}}{\xi^{2}}\right\} .
$$

In particular, at $v=0.0019$ and $\rho \approx 1 \mathrm{~cm}$, we have $\tilde{\sigma}_{\text {tot }}^{\perp} \approx 0.72 \mathrm{mb}$ and $\tilde{\sigma}_{\text {tot }}^{\|} \approx 0.81 \mathrm{mb}$. These values are not very different from each other and from $\sigma_{\text {tot }} \approx 0.75 \mathrm{mb}$ since a scale of the cross section is determined by values of radial integrals in the matrix element (5). Let us note that the contribution of the logarithmic term to the cross section at $|\xi| \gg 1$ is relatively small being about several percent in the above examples. If we neglect such terms, the dependence of the cross section on relative velocity is reduced to the common factor $\xi^{2}$.

We conclude that the cross section, which addresses the spin-flip transitions of an antiproton (proton) interacting with a polarized non-relativistic positron or electron is derived analytically. In the case of attraction, the cross section is strongly enhanced at $\alpha / v \gg 1$ as compared with that obtained in the Born approximation. However, this enhancement is insufficient for providing a practical use of positron beams to polarize stored antiprotons. Thus, the filtering method is still the most promising way for that (see, e.g. [10]).

[1] Technical Proposal for Antiproton-Proton Scattering Experiments with Polarization, PAX Collaboration, arXiv:hep-ex/0505054 (2005).

[2] F. Rathmann, Current status of the PAX project (9th PAX Meeting), Dubna, Russia, September 2005; available from the PAX website at http://www.fz-juelich.de/ikp/pax.

[3] A.I. Milstein and V.M. Strakhovenko, Phys. Rev. E 72 (2005) 066503.

[4] N.N. Nikolaev and F.F. Pavlov, hep-ph/0601184.

[5] L.D. Landau, E.M. Lifshits, Quantum Mechanics, Nonrelativistic Theory, Pergamon, Oxford (1965).

[6] Th. Walcher, H. Arenhövel, K. Aulenbacher, R. Barday, A. Jankowiak, Eur. Phys. J. A 34 (2007) 447.

[7] H. Arenhövel, Eur. Phys. J. A 34 (2007) 303.

[8] V.B. Berestetski, E.M. Lifshits, and L.P. Pitaevsky, Quantum Electrodynamics, Pergamon, Oxford (1982).

[9] A.I. Milstein, V.M. Strakhovenko, Phys. Lett. A 92 (1982) 381. 
[10] V.F. Dmitriev, A.I. Milstein, and V.M. Strakhovenko, Nucl. Instr. and Meth. B 266 (2008) 1122. 